\title{
TU/e emonownen

\section{Direct force-reflecting two-layer approach for passive bilateral teleoperation with time delays}

\section{Citation for published version (APA):}

Heck, D., Saccon, A., Beerens, R., \& Nijmeijer, H. (2018). Direct force-reflecting two-layer approach for passive bilateral teleoperation with time delays. IEEE Transactions on Robotics, 34(1), 194-206. [8268547].

https://doi.org/10.1109/TRO.2017.2769123

DOI:

10.1109/TRO.2017.2769123

Document status and date:

Published: 01/02/2018

\section{Document Version:}

Accepted manuscript including changes made at the peer-review stage

\section{Please check the document version of this publication:}

- A submitted manuscript is the version of the article upon submission and before peer-review. There can be important differences between the submitted version and the official published version of record. People interested in the research are advised to contact the author for the final version of the publication, or visit the $\mathrm{DOI}$ to the publisher's website.

- The final author version and the galley proof are versions of the publication after peer review.

- The final published version features the final layout of the paper including the volume, issue and page numbers.

Link to publication

\section{General rights}

Copyright and moral rights for the publications made accessible in the public portal are retained by the authors and/or other copyright owners and it is a condition of accessing publications that users recognise and abide by the legal requirements associated with these rights.

- Users may download and print one copy of any publication from the public portal for the purpose of private study or research.

- You may not further distribute the material or use it for any profit-making activity or commercial gain

- You may freely distribute the URL identifying the publication in the public portal.

If the publication is distributed under the terms of Article 25fa of the Dutch Copyright Act, indicated by the "Taverne" license above, please follow below link for the End User Agreement:

www.tue.nl/taverne

Take down policy

If you believe that this document breaches copyright please contact us at:

openaccess@tue.nl

providing details and we will investigate your claim. 


\title{
Direct force-reflecting two-layer approach for passive bilateral teleoperation with time delays
}

\author{
Dennis Heck, Alessandro Saccon, Ruud Beerens and Henk Nijmeijer
}

\begin{abstract}
We propose a two-layer control architecture for bilateral teleoperation with communication delays. The controller is structured with an (inner) performance layer and an (outer) passivity layer. In the performance layer, any traditional controller for bilateral teleoperation can be implemented. The passivity layer guarantees that, from the operator and environment perspective, the overall teleoperator is passive: the amount of energy that can be extracted from the teleoperator is bounded from below and the rate of increase of the stored energy in the teleoperator is bounded by (twice) the environment and operator supplied power. Passivity is ensured by modulating the performance layer outputs and by injecting a variable amount of damping via an energy-based logic that follows the innovative principle of energy duplication and takes into account the detrimental effects of time delays. In contrast to the traditional teleoperation approach, where the master and slave controllers implement an as-stiff-as-possible coupling between the master and slave devices, our scheme is specifically designed for direct force-reflecting bilateral teleoperation: the slave controller mimics the operator action, while the master controller reflects the slave-environment interaction. We illustrate the performance of the two-layer approach in a challenging experiment with a round-trip communication delay of $300 \mathrm{~ms}$ while making and breaking contact with a stiff aluminum environment. Finally, we also compare our controller with the state-of-the-art.
\end{abstract}

Index Terms-Bilateral control, telemanipulation, passivity, stability, time delay

\section{INTRODUCTION}

This work proposes a controller for direct force-reflection to guarantee passivity of a teleoperator in the presence of time delays. Numerous architectures have been proposed over the past thirty years to control bilateral teleoperators with communication delays (see [?], [?], [?] and the references therein). As depicted in ??, these can be classified in bilateral motion synchronization and direct force-reflection schemes.

In bilateral motion synchronization, both the master and slave controllers aim at motion synchronization. This class of architectures is characterized by a series connection of the teleoperator components. The environment force is reflected indirectly by creating a tight coupling between the master and slave (using, e.g., a virtual spring and damper), thereby reflecting the slave dynamics to the operator. A well known example is the position-position architecture [?], [?], [?]. As the time delays increase, guaranteeing stability while achieving

This research is supported by the Dutch Technology Foundation (STW, project 12157).

All authors are with the Eindhoven University of Technology, Department of Mechanical Engineering, Dynamics and Control group, P.O. Box 513, 5600 MB Eindhoven, the Netherlands (email: d.j.f.heck@tue.nl, a.saccon@tue.nl, h.nijmeijer@tue.nl).

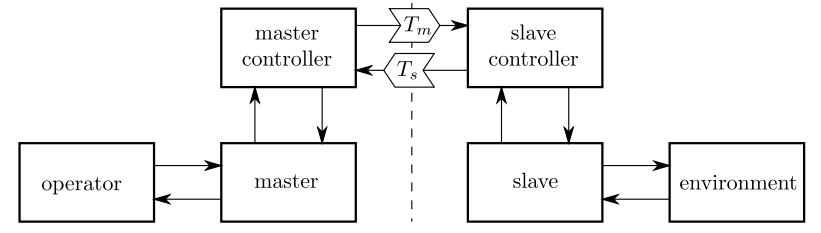

(a) Bilateral motion synchronization.

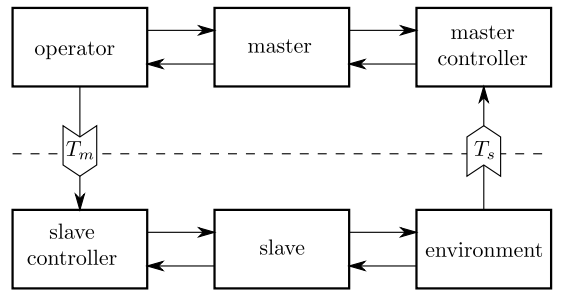

(b) Direct force-reflection.

Figure 1. Block diagrams of two different control approaches for bilateral teleoperation. Arrows represent information flows.

position tracking (i.e. asymptotic tracking), requires a reduction of the coupling strength; this holds independently of the specific implementation, be it the use of scattering [?], wave variables [?], [?], or damping injection [?], [?]. Moreover, "the lags between master and slave position movements cause large reaction forces to be supplied to the operator" [?]: These delay-induced forces, caused by the motion feedback terms in the master controller, can result in very high operator efforts in free motion.

In direct force-reflection, the slave controller acts as a virtual operator, and the master controller acts as a virtual environment. A well known architecture in this class is the position-force architecture [?], [?], [?]. When the slave is in free motion, the scheme is unilateral, and stability is not affected by the delays. Furthermore, the scheme is free of delay-induced forces or the reflection of the slave dynamics. Due to these benefits, direct force-reflecting architectures for delayed bilateral teleoperation seem to have more potential than bilateral motion synchronization in terms of force reflection, operator effort during free motion (related to injected damping) and motion synchronization. This was experimentally verified in [?, Chapter 3]. However, when the slave makes contact with the environment, the architecture switches from unilateral to bilateral. Even without delays this causes contact instabilities, as reported in, e.g., [?], [?], [?]. The contact instability manifests itself as a violent recoiling of the master device. The magnitude of this recoiling increases with the delay and the velocity of the slave device at impact, and is 
associated with active behavior of the teleoperator [?]. Due to the recoiling the operator is not able to make stable contact. Traditional approaches to solve this problem reduce the loop gain of the bilateral connection by canceling the induced master motion [?] or reflecting a projection of the environment force [?]. Unfortunately, the method presented in [?] is limited to linear systems, whereas the method presented in [?] is, in our opinion, prone to distort the operator's perception of the environment due to an anisotropic scaling of the environment force vector.

A common drawback of all mentioned approaches is that a significant and constant amount of damping, related to a worstcase situation, is injected to guarantee stability in the presence of delays. An effective and less conservative approach is to monitor the energy flows and adjust the control forces only when necessary. By using an energy conserving filter in the wave domain, [?] was one of the first papers proposing to exploit the monitoring of energy. Thereafter, the Time Domain Passivity Controller (TDPC) [?], [?] was proposed for architectures that do not use wave variables. TDPC continuously monitors the energy flows in the teleoperator and ensures passivity by adapting the damping gain. Ensuring passivity by modulating the reference, instead of the damping gain, is the corner stone of the passive-set-position-modulation (PSMP) approach, proposed in [?]. The method is suitable to be applied in bilateral motion synchronization schemes for teleoperation with time delays, showing, however, a substantial distortion of the contact forces (a factor 2) and relative positions of master and slave devices during a constrained motion phase. Other well-known control schemes that guarantee passivity in the context of bilateral motion synchronization are the Energy Bounding Algorithm (EBA), introduced in [?] and further developed in [?], and the Force Bounding Approach (FBA), introduced in [?]. Both EBA and FBA were initially developed and demonstrated for haptic applications, allowing for a passive coupling between an haptic device with a virtual environment.

In [?], the TDPC was restructured into a two-layer approach to explicitly separate the two goals of the teleoperator, i.e. achieving passivity and performance. In the inner Performance Layer (PeL) - actually named Transparency Layer in [?] - any existing architecture can be implemented. The forces computed by the PeL are then sent to the outer Passivity Layer (PaL). In [?], this layer enforces passivity by adapting the damping gain of the master controller to harvest energy from the operator. The harvested energy is stored in two energy tanks, one for each controller, and these controllers can only use the energy stored in these tanks. The distribution of the energy over the two tanks is achieved by synchronizing the available energy. Synchronizing the energy is required since the operator is the only source of energy for the controllers. Hence, the operator provides the energy required to move both the master and slave.

Synchronizing the energy appears to be natural for bilateral motion synchronization, but is not necessary for direct forcereflection. In this article, we therefore propose a two-layer architecture designed according to the direct force-reflection philosophy, i.e. where the slave and master controller represent a duplicate of the operator and the environment, respectively. In contrast to [?], the operator only supplies the energy required to actuate the slave device, because the environment supplies the energy to actuate the master device. Consequently, the novelty of our approach resides in the $\mathrm{PaL}$, where the master controller uses a duplicate of the energy transferred by the environment to the slave device and, similarly, the slave controller uses a duplicate of the energy transferred by the operator to the master device. The duplication of energy is based on the assumption that, from the perspective of the controllers, the master and slave devices are identical. Nonidentical master and slave devices can be virtually turned into identical devices by using a compensation, e.g. scaling, in a virtual control layer about each device. In our Pal, by monitoring the energy flows online, the damping gains of both controllers are adapted when active behavior is detected (recall that in [?] only the damping gain of the master controller is adapted). Moreover, when more energy is generated than allowed by the designer, the control force of the PeL is gradually reduced to prevent chattering.

This paper extends our previous work presented in [?], which contains a simulation study with a round-trip communication delay of $100 \mathrm{~ms}$ while the slave makes and breaks contact with an environment having a stiffness of 50,000 N/m. In contrast to [?], this paper contains proofs, experimental results with a round trip delay of $300 \mathrm{~ms}$ and a comparison with the state of the art 2-layer controller proposed in [?]. We stress to mention that although constant delays are assumed in our the analysis, the proposed controller can also be applied in case of bounded time-varying delays, by using, e.g., a buffer to make the delay appear constant.

We illustrate using an experimental 1-DOF setup that, for the challenging situation of having both a round-trip delay of $300 \mathrm{~ms}$ and a stiff aluminum environment, stable operation without a violent recoiling of the master is achieved when the proposed controller is employed. The variable damping gains are low when the slave is in free motion and high when the slave is in contact. This results in a minimal additional operator effort to move the teleoperator as the velocity during contact is almost zero, while stability in the contact phase is guaranteed. Without the PaL, the direct force-reflecting architecture is shown to perform rather poorly, as previously reported in, e.g., [?], [?] and [?]. Furthermore, an experimental comparison with the 2-layer controller proposed in [?] shows that the use of the controller proposed in this article requires significantly less damping during free motion and has improved transient responses during the impact and detachment phases.

This article is organized as follows. ?? explains the philosophy and the key details behind the proposed control scheme. The explicit controller design and model of the teleoperator are presented in ??. Passivity of the teleoperator is proven in ??. ?? details the model parameters presents the results of the experimental implementation. Conclusions are drawn in ??.

Notation. The subscripts $i \in\{m, s\}$ and $j \in\{m, s\}$, $j \neq i$, denote either the master $(m)$ or slave $(s)$ device. For the sake of brevity, we will often omit the explicit indication of time dependency: in particular, we will use $x$ and $x^{T}$ to denote, respectively, $x(t)$ and its delayed version $x(t-T)$. 
The dependency of an integrand on the integration variable is often also omitted.

\section{THE 2-LAYER ARCHITECTURE}

The foundations of the two-layer architecture detailed in this work will be illustrated by means of Figure 2. Therein, the teleoperator is highlighted by placing it into a light gray bounding box while the passivity layer is indicated with a dark gray background color. In the PeL, a traditional control algorithm is implemented to obtain optimal performance without considering passivity or stability. Ideally, this can be any force-reflecting algorithm, such as, e.g., a position-force controller. The PeL computes the desired forces $F_{m c} \in \mathbb{R}^{n}$ and $F_{s c} \in \mathbb{R}^{n}, n$ being the number of degrees of freedom, to actuate the master and slave, respectively, and these forces are sent to the PaL. The PaL, illustrated in dark gray in ??, monitors the power flows between several components of the teleoperator and is presented in detail below.

As mentioned in the introduction, the PeL used in the proposed controller plays the same role as the transparency layer in [?]. Our proposed PaL, however, is completely different since it neither requires the synchronization of energy nor must the operator supply the energy to move both the master and slave. In particular, our $\mathrm{PaL}$ is based on the philosophy of a direct force-reflecting architecture: the slave and master controllers represent, respectively, a virtual operator and environment. Seeing the master controller as a virtual environment leads to allow the PaL of the master controller to use a duplicate of the power $P_{e}:=-F_{e}^{\top} \dot{x}_{s}$ applied by the slave on the environment, with $F_{e} \in \mathbb{R}^{n}$ the force applied by the slave on the environment and $\dot{x}_{s} \in \mathbb{R}^{n}$ the slave velocity. Similarly, seeing the slave controller as a virtual operator leads to allow the PaL of the slave controller to use a duplicate of the power $P_{h}:=F_{h}^{\top} \dot{x}_{m}$ applied by the operator on the master, with $F_{h} \in \mathbb{R}^{n}$ the force applied by the operator and $\dot{x}_{m} \in \mathbb{R}^{n}$ the master velocity. Consequently, our PaL monitors the exchange of energy between the operator and the master, and the energy exchanged between the slave and the environment (in [?] the port between the master and master controller is monitored and compared with the port between the slave and slave controller).

Let $P_{h}$ and $P_{e}$ indicate the power inflow of the PaL and $P_{i c}:=F_{i c}^{\top} \dot{x}_{i}, i \in\{m, s\}$, the power ideally applied by the controller on the master and slave, as the power outflow. Denote with $E_{m, d i f f}$ and $E_{s, \text { diff }}$ the difference in the energy inflow and outflow of the controllers. Then, the rate of change of $E_{m, d i f f}$ and $E_{s, \text { diff }}$ are given by

$$
\begin{aligned}
\frac{d}{d t} E_{m, d i f f} & =P_{e}-P_{m c} \\
\frac{d}{d t} E_{s, d i f f} & =P_{h}-P_{s c} .
\end{aligned}
$$

Under ideal circumstances, assuming the master and slave devices to start at the exact same position and velocity with no communication delays, $P_{m c}=P_{e}$ and $P_{s c}=P_{h}$, such that $E_{i, d i f f}$ remains identically equal to zero when $F_{m c}=-F_{e}$ and $F_{s c}=F_{h}$ from the PeL are applied directly (recall that, as mentioned in the introduction, the master and slave dynamics are assumed to be identical, or made approximately so by a virtual layer).

In practice, such an ideal situation occurs rarely due to differences in the master and slave dynamics and the existence of delays, so the control forces $F_{m c}$ and $F_{s c}$ will in general not result in $P_{m c} \equiv P_{e}$ and $P_{s c} \equiv P_{h}$. As a consequence, both $E_{m, \text { diff }}$ and $E_{s, \text { diff }}$ will, if left uncontrolled, diverge from zero. Controlling $E_{i, d i f f}$ to zero is the novelty of the proposed controller and is the task assigned to the proposed PaL. The $\mathrm{PaL}$ is identical for both the master and slave controller, so for illustration purposes, this section focuses only on the description of the slave controller, that should be seen as a virtual operator.

A positive value of $E_{s, \text { diff }}$ means that the slave controller applied less energy to actuate the slave than the operator applied to control the master, whereas a negative value of $E_{s, \text { diff }}$ implies that the slave controller used more energy. The latter, $E_{s, \text { diff }}<0$, is associated with undesired active behavior of the controller. In [?], to prevent active behavior, the control force is cut off completely, such that the energy tanks have a lower bound at zero. In contrast, our controller allows bounded active behavior to reduce the performance loss in such a situation. To guarantee boundedness of the active behavior, the $\mathrm{PaL}$ requests extra power from the master device, the signal $P_{s, g e n}$ in ??, to increase the energy level $E_{s, d i f f}$. The requested energy is harvested by injecting a variable amount of damping on the master device, indicated by $F_{m \text {,harv }}$. The variable damping gain increases as the magnitude of $E_{m, h a r v}$, the amount of energy that must still be harvested from the master, increases.

In case the amount of harvested energy is insufficient to compensate for the energy shortage in the slave controller, e.g., due to zero velocity of the master, and $E_{s, b a l}$, the total energy balance of the slave $\mathrm{PaL}$, introduced later on in (??), drops below a designed threshold $E_{s, b 1}<0$, the control force $F_{s c}$ coming from the PeL is gradually decreased. This is done by modulating $F_{s c}$ with a continuous and state-dependent variable gain $\lambda_{s}\left(\underline{E}_{s, b a l}\right) \in[0,1]$. Here, $\underline{E}_{s, b a l}$ is a lower bound of $E_{s, b a l}$ that can be computed online and is defined later on in (??). The gain $\lambda_{s}\left(\underline{E}_{s, b a l}\right)$ is equal to one if $\underline{E}_{s, b a l} \geq E_{s, b 1}$. If $E_{s, b 1}>\underline{E}_{s, b a l}>E_{s, b 2}, \lambda_{s}\left(\underline{E}_{s, b a l}\right)$ monotonically decreases from one to zero for decreasing values of $\underline{E}_{s, b a l}$. An example of such a function is illustrated in ??. In the extreme case when $\underline{E}_{s, b a l} \leq E_{s, b 2}$, the variable gain $\lambda_{s}$ is set to zero, such that the slave controller can no longer apply energy to the slave device. Additionally, when $\underline{E}_{s, b a l}<E_{s, b 1}$ the PaL recovers from the energy shortage by applying a force $\left(1-\lambda_{s}\right) F_{s, r e c}$ with a variable amount of damping on the slave device.

The design of the bounds $0>E_{s, b 1}>E_{s, b 2}$ affect how aggressively the $\mathrm{PaL}$ responds to an energy shortage in the controller. When $\underline{E}_{s, b a l}>E_{s, b 1}$, the variable gain $\lambda_{s}$ equals one, such that the PeL force $F_{s c}$ is applied without modification. A more negative value of $E_{s, b 1}$ allows for a wider range of temporarily active behavior. A small difference between $E_{s, b 1}$ and $E_{s, b 2}$ implies an aggressive fluctuation of $\lambda_{s}$, while a larger difference creates a more gradual fluctuation.

Summarizing, the PaL of the slave controller monitors and 


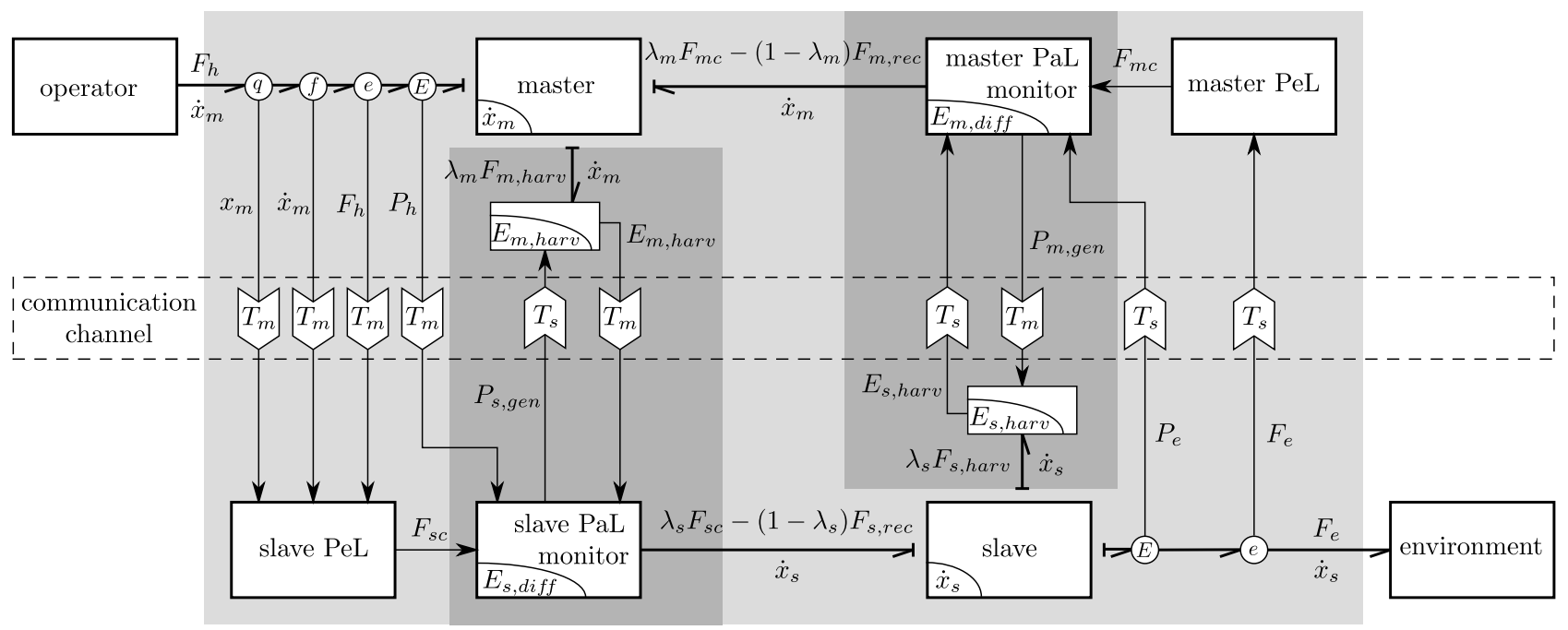

Figure 2. Bond graph of the proposed 2-layer architecture. The teleoperator is indicated with a light gray background color, the Passivity Layer with a dark gray background color. A half arrow indicates a bi-directional exchange of physical energy (power bond) and a full arrow represents a measurement (signal bond). The symbols $E, e, f$ and $q$ represent, respectively, power-, effort-, flow- and position sensors.

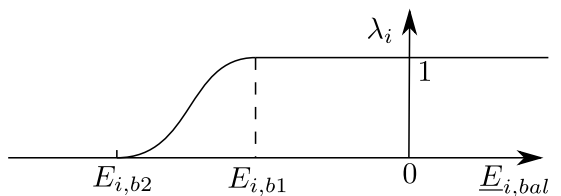

Figure 3. The state-dependent variable gain $\lambda_{i}$ as a function of $\underline{E}_{i, b a l}$.

regulates the use of a duplicate of the energy applied by the operator in order to apply a force $F_{s c}$ coming from the PeL. When a mismatch between the energy delivered by the slave controller and the energy supplied by the operator is detected, two scenarios are possible. In the first, when the controller uses less energy than the operator, part of the excess energy is discarded, because the slave does not need it. In the second, when the PeL force requires more energy than the operator, more energy is asked from the operator by injecting a variable amount of damping on the master. If insufficient energy is harvested from the master, e.g., because the master is moving slowly, energy is harvested from the slave and the PeL force $F_{s c}$ is modulated.

The same design is used for the $\mathrm{PaL}$ of the master controller. The only difference is that the master controller represents the virtual environment, so the $\mathrm{PaL}$ of the master controller uses a duplicate of the energy applied by the environment. If there is insufficient energy to apply the PeL force $F_{m c}$, extra energy is harvested by injecting a variable amount of damping on the slave. If not enough energy can be harvested from the slave, the energy is harvested from the master and the PeL force $F_{m c}$ is modulated.

\section{SYSTEM MODELING AND CONTROLLER DESIGN}

This section presents the considered teleoperator dynamics and further detailing of the PaL illustrated in ??.

\section{A. Teleoperator model}

It is assumed that gravity is compensated in the controllers, and that the master and slave dynamics, described in Cartesian space by

$$
\begin{aligned}
M_{m}\left(x_{m}\right) \ddot{x}_{m}+C_{m}\left(x_{m}, \dot{x}_{m}\right) \dot{x}_{m} & \\
+f_{m}\left(x_{m}, \dot{x}_{m}\right) & =F_{h}+F_{m c}^{*}, \\
M_{s}\left(x_{m}\right) \ddot{x}_{s}+C_{s}\left(x_{s}, \dot{x}_{s}\right) \dot{x}_{s}+f_{s}\left(x_{s}, \dot{x}_{s}\right) & =F_{s c}^{*}-F_{e},
\end{aligned}
$$

hold globally. In (??)-(??), $x_{i} \in \mathbb{R}^{n}$ is the end-effector position of device $i, M_{i}\left(x_{i}\right)>0 \in \mathbb{R}^{n \times n}$ the inertia matrix, $C_{i}\left(x_{i}\right)>0 \in \mathbb{R}^{n \times n}$ the Coriolis' matrix and $f_{i}\left(x_{i}, \dot{x}_{i}\right) \in \mathbb{R}^{n}$ the friction force. The inputs $F_{h}$ and $F_{e}$, as mentioned in ??, are the operator and environment forces. The control forces $F_{m c}^{*}$ and $F_{s c}^{*}$ are the output of the PaL. Details about how they are computed are presented in the next subsection, after the introduction of the following two assumptions, used in ?? to prove passivity of the teleoperator.

Assumption 1: The input forces $F_{h}$ and $F_{e}$ satisfy

$$
\begin{aligned}
& \text { 1) }\left\|F_{h}\right\| \leq \bar{F}_{h} \text { and }\left\|F_{e}\right\| \leq \bar{F}_{e} \text {, with } \bar{F}_{h}, \bar{F}_{e}>0 \text {, } \\
& \text { 2) } F_{h}^{\top} \dot{x}_{m} \leq 0 \text {, if }\left|\dot{x}_{m}\right|>\dot{x}_{m}^{a}>0 \text {, and } \\
& \text { 3) }-F_{e}^{\top} \dot{x}_{s} \leq 0 \text {, if }\left|\dot{x}_{s}\right|>\dot{x}_{s}^{a}>0 \text {. }
\end{aligned}
$$

Condition 1 of ?? implies that the forces applied by the operator and environment are bounded. Boundedness of $F_{h}$ is plausible due to the operator's physical limitations. Conditions 2 and 3 allow for an active operator and environment as long as the master and slave velocities do not exceed $\dot{x}_{i}^{a}$. As a result, ?? allows for both passive and bounded active behavior as long as $\dot{x}_{i}$ is smaller than an arbitrarily large constant $\dot{x}_{i}^{a}$. It is therefore somehow less restrictive than the passive operator and environment assumption that is typically required to guarantee passivity or stability (see e.g. [?] and the references therein). Strictly speaking, however, not every imaginable passive system will satisfy Assumption 1 and for those systems our passivity result cannot be applied.

Assumption 2: The control forces $F_{i c}$ of the PeL satisfy 
1) $\left\|F_{i c}\right\| \leq \bar{F}_{i c}$, with $\bar{F}_{i c}>0$, and

2) $F_{i c}^{\top} \dot{x}_{i} \leq 0$, if $\left|\dot{x}_{i}\right|>\dot{x}_{i}^{b}>0$.

?? implies that the PeL forces $F_{i c}$ are bounded and do not inject energy in the master and slave device above a certain velocity $\dot{x}_{i}^{b}$, which can be selected arbitrarily large to not affect stable operation. ?? can be satisfied by using a (smooth) saturation function to modify $F_{i c}$ whenever ?? would be violated otherwise.

\section{B. Design of the Passivity Layer controller}

As described in words in ??, the PaL control force $F_{i c}^{*}$ applied on device $i$ consists of the PeL force $F_{i c}$, modulated by $\lambda_{i} \in[0,1]$, and the harvesting and recovering forces $F_{j, \text { harv }}$ and $F_{i, \text { rec }}$. Namely,

$$
F_{i c}^{*}=\lambda_{i} F_{i c}-\lambda_{i} F_{i, h a r v}-\left(1-\lambda_{i}\right) F_{i, r e c} .
$$

The harvesting and recovery forces need to satisfy $\lambda_{j} F_{j, \text { harv }}^{\top} \dot{x}_{j} \geq 0$ and $\left(1-\lambda_{i}\right) F_{i, \text { rec }}^{\top} \dot{x}_{i} \geq 0$ (recall that $j \in\{m, s\}, j \neq i$ ). This work considers

$$
\begin{aligned}
F_{j, \text { harv }} & =-\beta_{j} E_{j, \text { harv }} \dot{x}_{j}, \\
F_{i, \text { rec }} & =-\gamma_{i} \underline{E}_{i, \text { bal }} \dot{x}_{i},
\end{aligned}
$$

but other choices are, in principle, also possible. In (??) and (??), the gains $\beta_{j}$ and $\gamma_{i}$ are strictly positive, such that the terms $-\beta_{j} E_{j, h a r v} \geq 0$ and $-\left(1-\lambda_{i}\right) \gamma_{i} \underline{E}_{i, \text { bal }} \geq 0$ in (??) represent variable damping gains, which increase for decreasing values of $E_{j, h a r v} \leq 0$ and $\underline{E}_{i, b a l}$, respectively. In (??), $E_{j, h a r v}$ represents the amount of energy that must still be harvested from device $j$ (see ??), as explained in ??. Furthermore, $\underline{E}_{i, b a l}$, defined later on in (??), is a conservative but online available lower bound of $E_{i, b a l}$, the energy balance of the PaL controller. In details, the balance $E_{i, b a l}$ consists of $E_{i, d i f f}$ on the local side of the controller, the energy $E_{j, h a r v}$ on the remote side of the controller, and the energy stored in the communication channel obtained by integrating the power request $-P_{i, g e n}$ over the delay interval:

$$
E_{i, b a l}:=E_{i, d i f f}+E_{j, h a r v}-\int_{t-T_{i}}^{t} P_{i, g e n} d \tau .
$$

In (??), $T_{i}$ represents, depending on the value of $i, T_{m}$ or $T_{s}$, the delays from master to slave and from slave to master, respectively.

Using (??) and including the effect of the delays, the evolution of the energy levels $E_{i, d i f f}$, presented in a simplified form in (??), is given by

$$
\begin{aligned}
\dot{E}_{m, d i f f}= & P_{e}^{T_{s}}+P_{m, g e n}-\lambda_{m} F_{m c}^{\top} \dot{x}_{m}-\lambda_{m} P_{m, d i s s} \\
& +\left(1-\lambda_{m}\right) F_{m, r e c}^{\top} \dot{x}_{m} \\
\dot{E}_{s, d i f f}= & P_{h}^{T_{m}}+P_{s, g e n}-\lambda_{s} F_{s c}^{\top} \dot{x}_{s}-\lambda_{s} P_{s, d i s s} \\
& +\left(1-\lambda_{s}\right) F_{s, r e c}^{\top} \dot{x}_{s}
\end{aligned}
$$

where the dissipation $P_{i, \text { diss }}$, active for $E_{i, \text { diff }}>0$, equals

$$
P_{i, d i s s}= \begin{cases}0, & \text { if } E_{i, d i f f}<0, \\ \alpha_{i} E_{i, d i f f}, & \text { if } E_{i, d i f f} \geq 0\end{cases}
$$

with $\alpha_{i}>0$ a design parameter. The dissipation $P_{i, \text { diss }}$ prevents that $E_{i, \text { diff }}$ grows unbounded. Without $P_{i, \text { diss }}$, this could occur in one of the two scenarios mentioned in ?? where the slave uses less energy than the operator. In [?], this growth is referred to as an energy build-up in the controller, which can substantially delay the reaction of the PaL to unstable behavior.

When $E_{i, d i f f}<0$, power is generated immediately by

$$
P_{i, g e n}= \begin{cases}-\alpha_{i} E_{i, d i f f} & \text { if } E_{i, d i f f}<0, \\ 0 & \text { if } E_{i, d i f f} \geq 0 .\end{cases}
$$

Note that as $P_{i, \text { diss }} \geq 0$ and $P_{i, g e n} \geq 0$, there is a persistent attempt to steer $E_{i, \text { diff }}$ towards zero, which is the key principle of the PaL. The amount of generated power $P_{i, g e n}$ on the local side is sent to the remote side, and used as the input for the harvesting dynamics

$$
\dot{E}_{j, h a r v}=\lambda_{j} F_{j, h a r v}^{\top} \dot{x}_{j}-P_{i, g e n}^{T_{i}}
$$

with $F_{j, \text { harv }}$ given in (??). Due to the first order dynamics (??), $E_{j, h a r v}$ is guaranteed to remain negative, because $P_{i, g e n} \geq 0$ and, by design, $\lambda_{j} F_{j, h a r v}^{\top} \dot{x}_{j} \geq 0$.

The time derivative of (??), using (??) and (??), is

$$
\begin{aligned}
\dot{E}_{m, \text { bal }}= & P_{e}^{T_{s}}-\lambda_{m} F_{m c}^{\top} \dot{x}_{m}+\lambda_{s} F_{s, \text { harv }}^{\top} \dot{x}_{s} \\
& -\lambda_{m} P_{m, \text { diss }}+\left(1-\lambda_{m}\right) F_{m, r e c}^{\top} \dot{x}_{m}, \\
\dot{E}_{s, \text { bal }}= & P_{h}^{T_{m}}-\lambda_{s} F_{s c}^{\top} \dot{x}_{s}+\lambda_{m} F_{m, h a r v}^{\top} \dot{x}_{m} \\
& -\lambda_{s} P_{s, \text { diss }}+\left(1-\lambda_{s}\right) F_{s, \text { rec }}^{\top} \dot{x}_{s} .
\end{aligned}
$$

Due to the delays, $E_{i, b a l}$ in (??) cannot be computed online as $\lambda_{j} F_{j, h a r v}^{\top} \dot{x}_{j}$ at time $t$ is not available to controller $i$. However,

$$
\begin{aligned}
E_{j, \text { harv }} & =E_{j, \text { harv }}^{T_{j}}+\int_{t-T_{j}}^{t} \lambda_{j} F_{j, h a r v}^{\top} \dot{x}_{j} d \tau-\int_{t-T_{r}}^{t-T_{i}} P_{i, g e n} d \tau \\
& \geq E_{j, \text { harv }}^{T_{j}}-\int_{t-T_{r}}^{t-T_{i}} P_{i, \text { gen }} d \tau
\end{aligned}
$$

where $T_{r}:=T_{m}+T_{s}$ is the round-trip delay. Therefore, by defining

$$
\underline{E}_{i, b a l}:=E_{i, d i f f}+E_{j, h a r v}^{T_{j}}-\int_{t-T_{r}}^{t} P_{i, g e n} d \tau \leq E_{i, b a l},
$$

an online available lower bound of $E_{i, b a l}$ is readily obtained. Differentiating with respect to time the expression for $\underline{E}_{i, b a l}$ given in (??) and using (??) and (??), one obtains

$$
\begin{aligned}
\dot{E}_{m, \text { bal }}= & P_{e}^{T_{s}}-\lambda_{m} F_{m c}^{\top} \dot{x}_{m}+\lambda_{s}^{T_{s}}\left(F_{s, \text { harv }}^{T_{s}}\right)^{\top} \dot{x}_{s}^{T_{s}} \\
& -\lambda_{m} P_{m, \text { diss }}+\left(1-\lambda_{m}\right) F_{m, \text { rec }}^{\top} \dot{x}_{m}, \\
\dot{E}_{s, \text { bal }}= & P_{h}^{T_{m}}-\lambda_{s} F_{s c}^{\top} \dot{x}_{s}+\lambda_{m}^{T_{m}}\left(F_{m, \text { harv }}^{T_{m}}\right)^{\top} \dot{x}_{m}^{T_{m}} \\
& -\lambda_{s} P_{s, \text { diss }}+\left(1-\lambda_{s}\right) F_{s, \text { rec }}^{\top} \dot{x}_{s},
\end{aligned}
$$

which is similar to the expression for $E_{i, b a l}$ in (??).

Summarizing, the teleoperator consists of the device dynamics (??)-(??) and the controller defined by (??)-(??), (??)-(??) and (??). Its inputs are $F_{h}$ and $-F_{e}$ and its outputs $\dot{x}_{m}$ and $\dot{x}_{s}$. It is assumed that ???? hold. In the following section it is proven that the proposed teleoperator is passive. 


\section{PASSIVITY OF THE TELEOPERATOR}

We provide here a proof of passivity for the teleoperator detailed in ??. To this end, we define the total energy of the teleoperator (the light gray box in ??) to be the storage functional $V$ which consists of the energy $E_{i}:=\frac{1}{2} \dot{x}_{i}^{\top} \bar{M}_{i}\left(x_{i}\right) \dot{x}_{i}$ of the master and slave devices, the energy $E_{i, b a l}$ present in the PaL, given by (??), and the energies $E_{h, c o m}:=\int_{t-T_{m}}^{t} P_{h} d \tau$ and $E_{e, c o m}:=-\int_{t-T_{s}}^{t} P_{e} d \tau$ stored in the communication channel due to the power duplication:

$$
V:=E_{m}+E_{s}+E_{m, b a l}+E_{s, b a l}+E_{h, c o m}+E_{e, c o m} .
$$

The energies $E_{m}$ and $E_{s}$ satisfy

$$
\begin{gathered}
\dot{E}_{m}=F_{h}^{\top} \dot{x}_{m}+\lambda_{m} F_{m c}^{\top} \dot{x}_{m}-\lambda_{m} F_{m, h a r v}^{\top} \dot{x}_{m} \\
-\left(1-\lambda_{m}\right) F_{m, r e c}^{\top} \dot{x}_{m} \\
\dot{E}_{s}=-F_{e}^{\top} \dot{x}_{s}+\lambda_{s} F_{s c}^{\top} \dot{x}_{s}-\lambda_{s} F_{s, h a r v}^{\top} \dot{x}_{s} \\
-\left(1-\lambda_{s}\right) F_{s, r e c}^{\top} \dot{x}_{s}
\end{gathered}
$$

???? imply both $E_{i}$ are bounded:

Property 1: Given ????, consider the closed loop dynamics of (??)-(??) using the controller (??). At the initial time $t_{0}$, let $\left|\dot{x}_{i}\left(t_{0}\right)\right| \leq \overline{\dot{x}}_{i}:=\max \left(\dot{x}_{i}^{a}, \dot{x}_{i}^{b}\right)<\infty$. Then, the energy $E_{i}=\frac{1}{2} \dot{x}_{i}^{\top} M_{i}\left(x_{i}\right) \dot{x}_{i}$ satisfies for all $t \geq t_{0}$

$$
0 \leq E_{i} \leq \bar{E}_{i}=: \frac{1}{2} \nu_{i} \overline{\dot{x}}_{i}^{2}
$$

with $\nu_{i}$ the largest eigenvalue of $M_{i}\left(x_{i}\right)$.

Proof: At time $t_{0},\left|\dot{x}_{i}\left(t_{0}\right)\right| \leq \overline{\dot{x}}_{i}$, and since $M_{i}\left(x_{i}\right) \leq$ $\nu_{i} I$ (see [?, Chapter 7]), with $I$ the $n \times n$ identity matrix, it follows that $E_{i}\left(t_{0}\right) \leq \bar{E}_{i}$. Suppose that a time $t^{*}$, where $\left|\dot{x}_{i}\right|=\overline{\dot{x}}_{i}$, does not exist. Then $\left|\dot{x}_{i}\right|<\overline{\dot{x}}_{i}$ for all $t \geq t_{0}$ and it follows directly that $E_{i}<\frac{1}{2} \overline{\dot{x}}_{i}^{\top} M_{i}\left(x_{i}\right) \overline{\dot{x}}_{i} \leq \frac{1}{2} \nu_{i} \overline{\dot{x}}_{i}^{2}=\bar{E}_{i}$ for all $t \geq t_{0}$. If such a time $t^{*}$, where $\left|\dot{x}_{i}\right|=\overline{\dot{x}}_{i}$, does exist, then $E_{i}\left(t^{*}\right)=\frac{1}{2} \overline{\dot{x}}_{i}^{\top} M_{i}\left(x_{i}\right) \overline{\dot{x}}_{i} \leq \frac{1}{2} \nu_{i} \overline{\dot{x}}_{i}^{2}=\bar{E}_{i}$. The first two terms of (??) at $t^{*}$ satisfy $F_{h}^{\top} \dot{x}_{m} \leq 0$ and $-F_{e}^{\top} \dot{x}_{s} \leq 0$ due to ??, and $F_{i c}^{\top} \dot{x}_{i} \leq 0$ due to ??. The last two terms on the right hand side of (??) are nonpositive by design. As a result, $\dot{E}_{i}\left(t^{*}\right) \leq 0$, and due to the first order dynamics of (??) it follows that $E_{i} \leq \bar{E}_{i}$ is an invariant set, such that $E_{i} \leq \bar{E}_{i}$ for all $t \geq t_{0}$. Finally, the lower bound of $E_{i}$ at 0 follows from $M_{i}\left(x_{i}\right)>0$, which completes the proof.

The following theorem is one of the key results of this paper.

Theorem 1: Given ????, consider the teleoperator described by (??), (??), (??)-(??), (??)-(??), (??), having input $u:=$ $\left[F_{h} ;-F_{e}\right]$ and output $y:=\left[\dot{x}_{m} ; \dot{x}_{s}\right]$. At initial time $t_{0}$, let $\left|\dot{x}_{i}\left(t_{0}\right)\right| \leq \overline{\dot{x}}_{i}$ and $\underline{E}_{i, b a l}\left(t_{0}\right)>E_{i, b 2}$, with $E_{i, b 2}$ introduced in ??. Then, for all $t \in\left[t_{0}, \infty\right)$, the storage functional $V$, defined in (??), satisfies

$$
\begin{aligned}
V & >\underline{V}>-\infty \\
\frac{d V}{d t} & \leq 2 u^{\top} y
\end{aligned}
$$

with

$$
\underline{V}:=-\bar{E}_{m}-\bar{E}_{s}-\Delta \bar{E}_{e}-\Delta \bar{E}_{h}+E_{m, b 2}+E_{s, b 2}+H,
$$

$H:=\min \left(E_{m, b 2}-\alpha_{m} \bar{E}_{m}-E_{m, b 3}-E_{m, \operatorname{diff}}\left(t_{0}\right)-\right.$ $\left.2 \Delta \bar{E}_{e}, E_{s, b 2}-\alpha_{s} \bar{E}_{s}-E_{s, b 3}-E_{s, \text { diff }}\left(t_{0}\right)-2 \Delta \bar{E}_{h}\right), \bar{E}_{i}$ given in (??), and the constant

$$
\begin{gathered}
E_{i, b 3}:=\left(\bar{F}_{h} \dot{x}_{m}^{a}+\bar{F}_{e} \dot{x}_{s}^{a}\right) \frac{1}{\alpha_{i}} \\
\Delta \bar{E}_{h}:=\int_{t-T_{m}}^{t} \bar{F}_{h} \dot{x}_{m}^{a} d \tau, \quad \Delta \bar{E}_{e}:=\int_{t-T_{s}}^{t} \bar{F}_{e} \dot{x}_{s}^{a} d \tau .
\end{gathered}
$$

The bounds $\bar{F}_{h}, \bar{F}_{e}$ and $\dot{x}_{i}^{a}$ are given in ??.

Remark 1: Due to the presence of time delays, $V$ is a functional defined in terms of the (infinite dimensional) state of the teleoperator, not explicitly indicated here. Equation (??) in ?? states that the rate of change of the energy of the teleoperator is bounded from above by the power supplied by the operator and environment. The factor 2 in (??) is the result of the power duplication. Furthermore, equations (??) and (??) characterize $V$ as a storage functional and allow to conclude that the teleoperator is passive as $V$ admits a lower bound. Note that (19) and (20) are equivalent to $\int u^{T} y d \tau \geq-\kappa$, with $\kappa:=0.5\left(V\left(t_{0}\right)-\underline{V}\right)$, retrieving the equivalent definition of passivity (with $\kappa$ depending on the initial conditions) found, e.g., in [3].

Proof: Equation (??) follows directly from ??, which is presented in the Appendix. To prove (??), differentiate $V$ in (??) with respect to time to obtain

$$
\dot{V}=\dot{E}_{m}+\dot{E}_{s}+\dot{E}_{m, b a l}+\dot{E}_{s, b a l}+\dot{E}_{h, c o m}+\dot{E}_{e, c o m},
$$

where $\dot{E}_{h, c o m}:=P_{h}-P_{h}^{T_{m}}$ and $\dot{E}_{e, c o m}:=P_{e}-P_{e}^{T_{s}}$ represent the power present in the communication channel due to the duplication. Then, (??) is obtained by using (??), and (??) in (??).

\section{EXPERIMENTS}

The purpose of this section is to demonstrate the effectiveness of the proposed controller when implemented on a physical setup. Previously, in [?], we presented illustrative simulation results with a round-trip communication delay of $100 \mathrm{~ms}$ while the slave makes and breaks contact with an environment having a stiffness of $50,000 \mathrm{~N} / \mathrm{m}$. In the experiments presented here, we consider a round-trip delay of $300 \mathrm{~ms}$ and the slave makes contact with an even stiffer environment, namely an aluminum cylinder. An accompanying video demonstrating the effectiveness of the proposed twolayer control approach over the lack of our passivity layer $(\mathrm{PaL})$ is available at http://ieeexplore.ieee.org.

Below, we first describe the employed physical setup and then present experiments with and without our proposed controller. Finally, we compare our controller with the stateof-the-art 2-layer controller proposed in [?].

\section{A. Experimental setup}

The experimental setup consists of the 1-DOF revolute master and slave devices depicted in ??. Each device is actuated with a Maxon RE 35 DC servo motor, which drives the rotational segment of the device with a capstan drive. The 


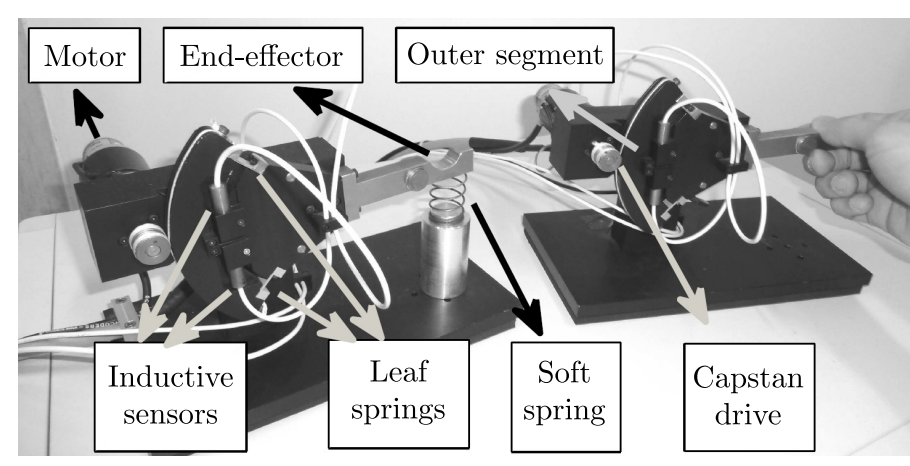

Figure 4. 1-DOF experimental setup (image adapted from [?]). Experiments are conducted with both a soft spring and a stiff aluminum cylinder.

rotation of the motor is measured with an incremental encoder, having a resolution of $2.1 \cdot 10^{-5} \mathrm{rad}$. The capstan drive has a reduction of $1 / 10$ and results in a resolution of $2.1 \cdot 10^{-6}$ rad for the rotational segment. This rotational segment has an operating range from $-0.73 \mathrm{rad}$ to $0.73 \mathrm{rad}$, with $0 \mathrm{rad}$ being the horizontal configuration. The operator or environment can interact with the end-effector, which is located at $7.5 \mathrm{~cm}$ from the point of rotation on the other side of this segment. The torque applied by the motor on the end-effector, after the capstan drive, is limited to $1.5 \mathrm{Nm}$.

The rotational segment is split in two concentric parts. These are connected by two short and thick leaf springs, having a known torsional stiffness of $3.5 \cdot 10^{3} \mathrm{Nm} / \mathrm{rad}$ [?]. The torque transmitted through the leaf springs is measured with two inductive sensors that measure the relative rotation between the inner and outer segment. The maximum difference in rotation is in the order of $100 \mu \mathrm{m}$. The resolution of the torque measurements is $5.25 \cdot 10^{-4} \mathrm{Nm}$. Note that the stiff force sensor measures the internal force of the link and not directly the environment force acting on the end-effector. Consequently, also payload forces of the inner segment are measured and transmitted to the other device (see e.g. ??). Fortunately, these forces are small compared to the contact forces and thus barely affect the operator's perception.

The proposed controller is implemented in Matlab Simulink ${ }^{\circledR}$ on a Linux-based computer. The sampling frequency is $1 \mathrm{kHz}$. The velocity signal is obtained by numerical differentiation of the position signal, in combination with a first-order lowpass filter with a cut-off frequency of $80 \mathrm{~Hz}$. The force signals are filtered with a first-order lowpass filter with a cut-off frequency of $15 \mathrm{~Hz}$.

For the considered setup, the dynamics (??)-(??) simplify to a 1-DOF mass-damper system, where the inertia $M_{i}$ and viscous friction $f_{i}\left(x_{i}, \dot{x}_{i}\right)=b_{i} \dot{x}_{i}$ are identified as $M_{m}=$ $2 \cdot 10^{-3} \mathrm{~kg} \mathrm{~m}^{2}, M_{s}=2.2 \cdot 10^{-3} \mathrm{~kg} \mathrm{~m}{ }^{2}, b_{m}=5 \cdot 10^{-3}$ $\mathrm{Nm}$ s/rad and $b_{s}=7 \cdot 10^{-3} \mathrm{Nm} \mathrm{s} / \mathrm{rad}$. The direct forcereflecting Position/Force-Force (PF-F) controller

$$
\begin{aligned}
F_{m c} & =-F_{e}^{T_{s}} \\
F_{s c} & =F_{h}^{T_{m}}+k_{p}\left(x_{m}^{T_{m}}-x_{s}\right)+k_{d}\left(\dot{x}_{m}^{T_{m}}-\dot{x}_{s}\right)
\end{aligned}
$$

is implemented in the PeL. In the experimental comparison presented in [?, Chapter 3], this controller is identified as the most suitable architecture to provide high performance in
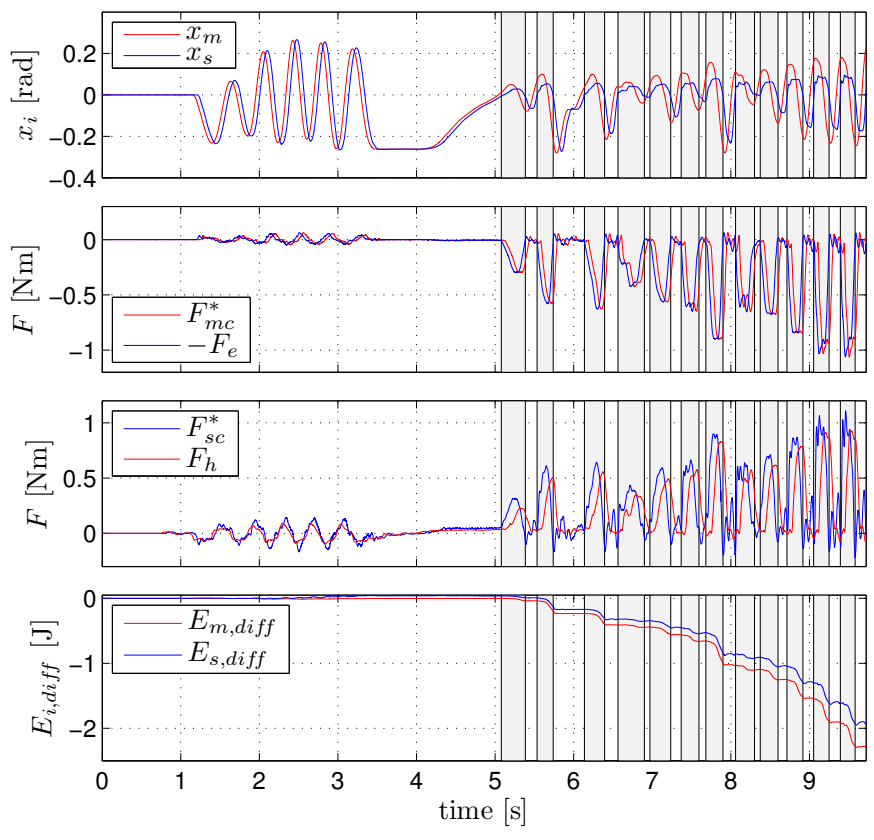

Figure 5. Experimental results without $\mathrm{PaL}$ for $T_{m}=T_{s}=0.05 \mathrm{~s}$ : Positions, forces and the energy difference $E_{i, d i f f}$ computed by (??). In the light gray area the slave is in contact with the spring.

both free motion and contact phases in the presence of delays. During all experiments, the proportional and derivative gains are selected as $k_{p}=11.2 \mathrm{Nm} / \mathrm{rad}$ and $k_{d}=0.089 \mathrm{Nm} \mathrm{s} / \mathrm{rad}$. Furthermore, the delays are $T_{m}=T_{s}=50 \mathrm{~ms}$, resulting in a round-trip delay of $T_{m}+T_{s}=100 \mathrm{~ms}$.

\section{B. Experimental results $T_{m}=T_{s}=0.05 \mathrm{~s}$ without the PaL}

To illustrate the effectiveness of the proposed controller, we first present two experiments, using a round-trip delay of only $100 \mathrm{~ms}$, in which the PaL is not used and the PeL controller (??) is implemented directly.

In the first experiment, the slave interacts with a spring (the"soft spring" in the center of ??), having stiffness $k_{e} \approx$ $2300 \mathrm{~N} / \mathrm{m}$. During the first 5 seconds of the results presented in ??, the slave is in free motion and follows the master with a delay $T_{m}=0.05 \mathrm{~s}$. After $5 \mathrm{~s}$ the operator attempts to keep the slave in contact with the spring. The light grey areas indicate when contact between the slave and the environment is detected by the force sensor. When the slave makes contact with the environment, the master recoils (see the introduction and [?], [?] for more background information regarding this phenomenon) and the operator is not able to keep the slave in contact with the spring, despite carefully approaching the surface. The slave bounces several times, with increasing amplitude, against the environment. Around $9.7 \mathrm{~s}$ the safety of the setup stops the experiment due to the high velocity of the master. Looking at the energy difference $E_{i, \text { diff }}$ computed by (??) (not used in the controller, since the PaL is inactive), it is observed that the teleoperator generates energy.

The recoiling of the master becomes even more violent when the slave interacts with an aluminum cylinder. The re- 

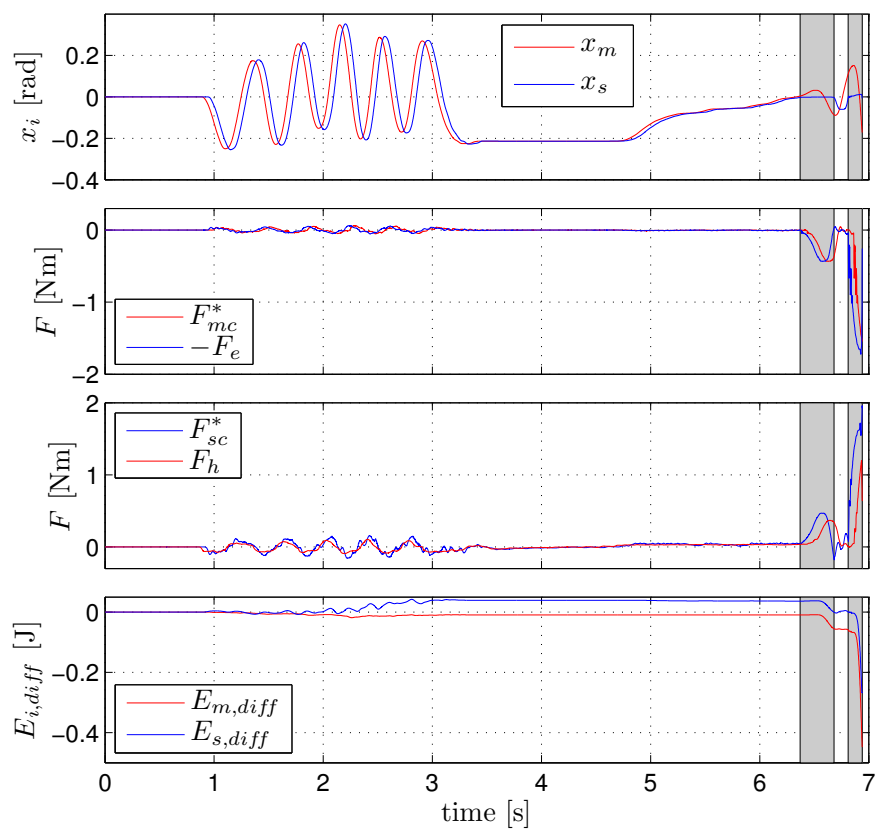

Figure 6. Experimental results without $\mathrm{PaL}$ for $T_{m}=T_{s}=0.05 \mathrm{~s}$ : Positions, forces and the energy difference $E_{i, \text { diff }}$ computed by (??). In the dark grey area the slave is in contact with the aluminum cylinder.

sults of this challenging task are shown in ??. The environment is approached carefully by the operator, nevertheless the slave bounces off the cylinder. The master recoils twice before the safety of the setup stops the experiment due to the too high master velocity. Again, the bouncing and recoiling are associated with active behavior ( $E_{i, d i f f}$ becomes negative) of the controller (??).

\section{Experimental results $T_{m}=T_{s}=0.15 \mathrm{~s}$ with the PaL}

Results for an experiment combining free motion (first $6 \mathrm{~s}$ ), contact with the spring (light grey area from about $6 \mathrm{~s}$ to $11 \mathrm{~s}$ ) and contact with the aluminum cylinder (dark grey area from about $15 \mathrm{~s}$ to $20 \mathrm{~s}$ ) with the proposed two-layer control architecture are shown in ??. To illustrate the stabilizing properties and achievable performance of our controller in realistic communication scenarios, the delay is increased compared to the experiments in the previous section to $T_{m}=T_{s}=0.15$ $\mathrm{s}$, resulting in a $300 \mathrm{~ms}$ round trip delay. The PaL parameters are set to $\alpha_{i}=5, \beta_{i}=15, \gamma_{i}=20, E_{i, b 1}=-15 \cdot 10^{-3}$ and $E_{i, b 2}=-8 \cdot 10^{-2}$ to minimally affect the response in free motion, but rapidly react to the impact and detachment of the slave-environment interaction. Due to a limited actuator torque, the upper bound of the control forces $F_{i c}$ in ?? is selected as $\bar{F}_{i c}=1.5 \mathrm{Nm}$ and the maximal allowed velocity is set to $\dot{x}_{i}^{b}=7 \mathrm{rad} / \mathrm{s}$. During the experiment the operator receives visual feedback (i.e. scopes on a display) on the applied force and master velocity and the operator is instructed to satisfy the same limits of the setup, i.e., in ??, $\bar{F}_{h}=1.5$ $\mathrm{Nm}$ and $\dot{x}_{m}^{a}=7 \mathrm{rad} / \mathrm{s}$. The environments considered here are passive.

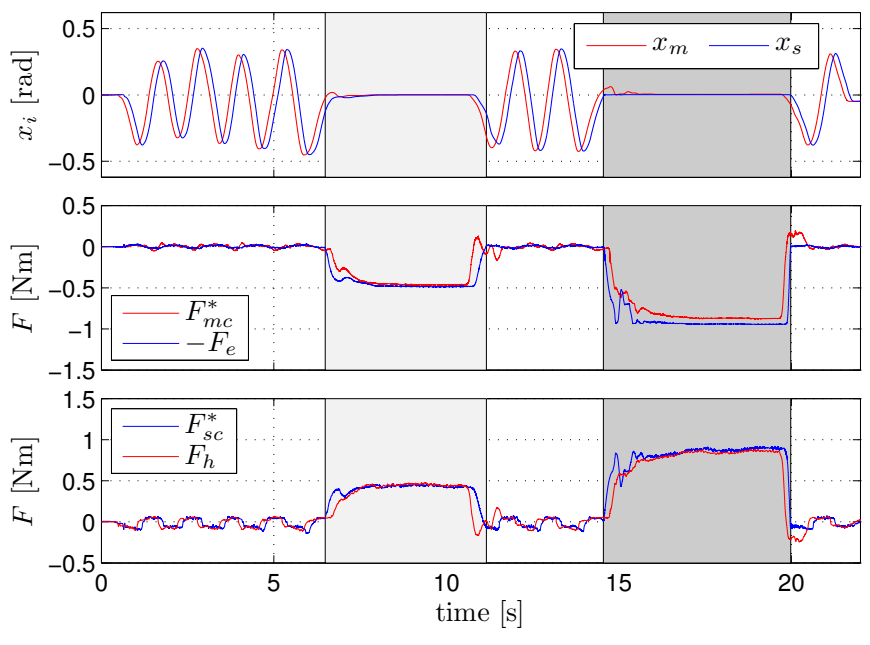

(a) Positions and forces.
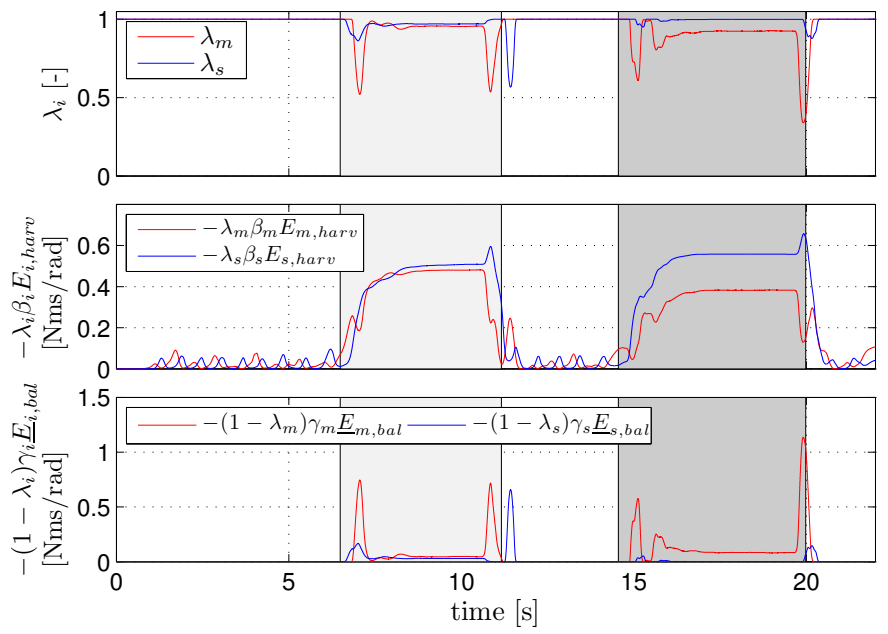

(b) Variable damping gains and $\lambda_{i}$.

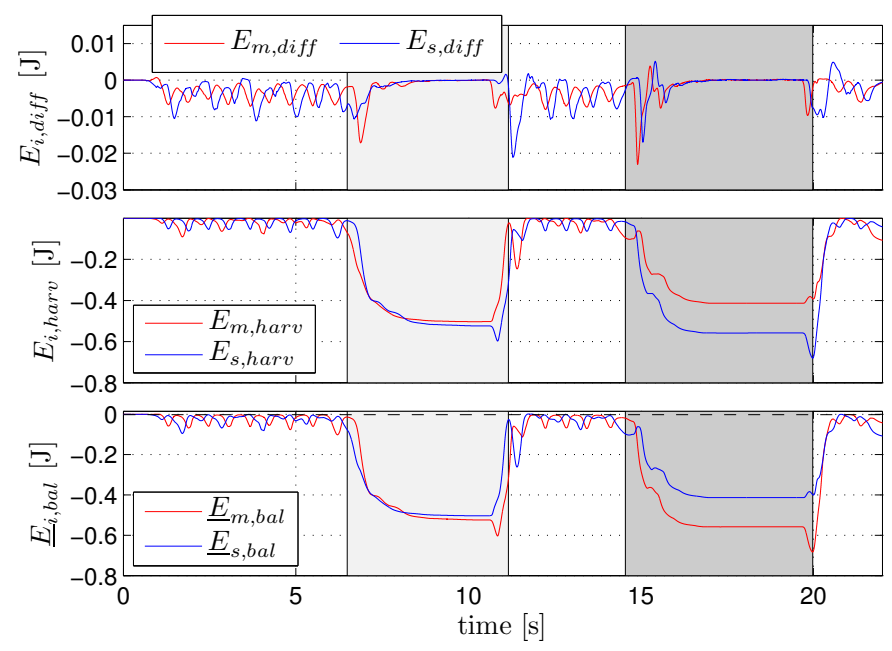

(c) Energy levels.

Figure 7. Experimental results with $\mathrm{PaL}$ for $T_{m}=T_{s}=0.15 \mathrm{~s}$. In the light grey area the slave is in contact with the spring $\left(x_{e}=-0.1 \mathrm{rad}\right)$. In the dark grey area the slave is in contact with the aluminum cylinder $\left(x_{e}=0 \mathrm{rad}\right)$. 
During free motion, the slave accurately follows the delayed motion of master despite the nonideality of the force measurement (i.e. the measured dynamics after the force sensor). Because the measured $F_{e}$ is applied to the master with a delay of $T_{s}=0.15 \mathrm{~s}$, the master has a different velocity than the slave. Consequently, the energy levels start to deviate from zero as shown in ??c (first 6 seconds) and the PaL becomes active. The controller gains of the $\mathrm{PaL}$ have been tuned such that the combined damping gains (the two lower plots of ??b) remain smaller than $0.06 \mathrm{Nms} / \mathrm{rad}$, and the resulting damping forces remain significantly smaller than the forces during contact. This allows the operator to detect the impact with the environment (according to the results presented in [?], having damping during free motion negatively affects the perception of hardness of the environment).

After contact of the slave with the spring, a small transient is present in both the motion and force profiles. This transient is the start of the recoiling of the master device due to the phenomenon described in [?]. Due to the PeL force $F_{m c}=-F_{e}^{T_{s}}$, the master is first slowed down and then pushed back, reversing its velocity. The associated active behavior is detected by the PaL. The variable damping gains increase and the variable force-reflection gains $\lambda_{i}$ are reduced to prevent a recoiling of the master, as occurred in ?? without PaL, and damp out the transient. After stable contact has been achieved, the operator is instructed to detach the slave from the spring (approximately at $10 \mathrm{~s}$ ). During the detachment phase the variable damping gains increase slightly, while simultaneously $\lambda_{i}$ decreases temporarily to prevent the master and slave to be accelerated from the environment due to the PeL force. Once the slave is in free motion, $\lambda_{i}$ quickly converges back towards 1 and the variable damping gains $\beta_{i} E_{i, h a r v}$ converge to zero.

A similar response of the master, slave and controller is obtained during contact of the slave with the aluminum cylinder. Compared to the contact phase with the spring, the oscillations following the impact have a larger amplitude, but the PaL eliminates them in about half a second. The variable damping gains and $\lambda_{i}\left(\lambda_{m}=0.92\right.$ and $\lambda_{s}=1$ during steadystate contact) are quite higher compared to the constant gains proposed in, e.g., [?], [?], indicating that the environment is perceived stiffer by the operator.

Summarizing, we have demonstrated experimentally that the proposed two-layer architecture is capable of achieving stable contact for a $300 \mathrm{~ms}$ round trip delay and that it is also robust to imperfect velocity and force measurements, and setup parameters. In free motion, the damping gains are low and, consequently, $\lambda_{i}$ is almost always equal to one. When the slave makes contact with the environment, the PaL prevents active behavior, in particular, the dangerous recoiling of the master during the impact and detachment phase typically observed when employing the standard position-force control in delayed teleoperation. The prevention of this recoiling is achieved by temporarily increasing the variable damping gains and slightly reducing the force-reflection gains $\lambda_{i}$. The adaptation of the damping- and force-reflection gains occurs automatically as a consequence of the monitoring and control of the energy duplication in the PaL. Consequently, the performance loss during the free motion, impact, contact and detachment phases is rather limited. Interestingly, the results presented in [?] suggest that increasing the damping only during the contact phase, so having low damping gains during free motion and high damping gains during contact, has a positive effect on the perception of hardness of the environment. This could partially compensate for the negative effect of reducing $\lambda_{i}$ on the perception of the environment.

\section{Comparison with the 2-layer controller presented in [?]}

This section presents an experimental comparison with the 2-layer controller proposed in [?]. As mentioned earlier, the Transparency Layer (TL) of [?] is similar to our PeL. Hence, for the comparison, we use the PF-F controller (??) in the TL.

In [?], their $\mathrm{PaL}$ guarantees passivity of the teleoperator by controlling the energy applied by both controllers, but the main difference compared to our PaL is that a different and single energy balance is considered. To maintain this energy balance, a variable damping force is applied only by the master controller to harvest energy from the operator. Without going into details (see [?] for more information), the energy balance is expressed by two energy tanks, one for each controller, with energy levels $H_{i}$ representing the maximum energy the controllers are allowed to apply on the master and slave devices. The energy level of the master tank is controlled to a desired tank level $H_{d}$ by the Tank Level Controller (TLC) by adding a damping force $F_{T L C}$ to the master force $F_{m c}$ of the TL

$$
\bar{F}_{m c}=F_{m c}+F_{T L C} .
$$

The damping force is defined as

$$
F_{T L C}=-\max \left(d\left(H_{d}-H_{m}\right), 0\right) \dot{x}_{m},
$$

where $d$ is a tuning parameter and the max-operator sets the damping gain to zero whenever the energy of the master exceeds the desired level $\left(H_{m}>H_{d}\right)$. Since the TLC only regulates $H_{m}$ to $H_{d}$, and not $H_{s}$, in [?] both energy levels $H_{i}$ are synchronized by sending a fraction $\delta_{i}$ of $H_{i}$ to the other tank $H_{j}$.

For the experiments we performed with the controller proposed in [?], we used $H_{d}=0.05 \mathrm{~J}, d=20 \mathrm{Ns} / \mathrm{Jm}$ and $\delta_{i}=0.01$. The experimental results are shown in ??. In particular, ??a shows the same signals as ??a for our proposed PaL, only the PaL control forces are denoted by $\bar{F}_{i c}$ (note that $\bar{F}_{s c}=F_{s c}$ ). ??b shows the energy levels $H_{i}$ of the tanks, the variable damping gain $d\left(H_{d}-H_{m}\right)$ and TLC force computed by (??). At a quick look, the response appears to be similar to the response obtained with our 2-layer controller (compare ??a with ??a). There are, however, relevant differences during the free motion, impact, contact and detachment phases. These differences are as follows. Compared to our controller, the controller proposed in [?] has

1) significantly higher damping gains and forces during the free motion phase (compare bottom plots ??b with bottom plots ??b) and are most dominant during velocity reversals. This is the result of using only one energy balance (master and slave tanks are connected) and injecting variable damping only on the master side to prevent active behavior of both the master and slave device. 

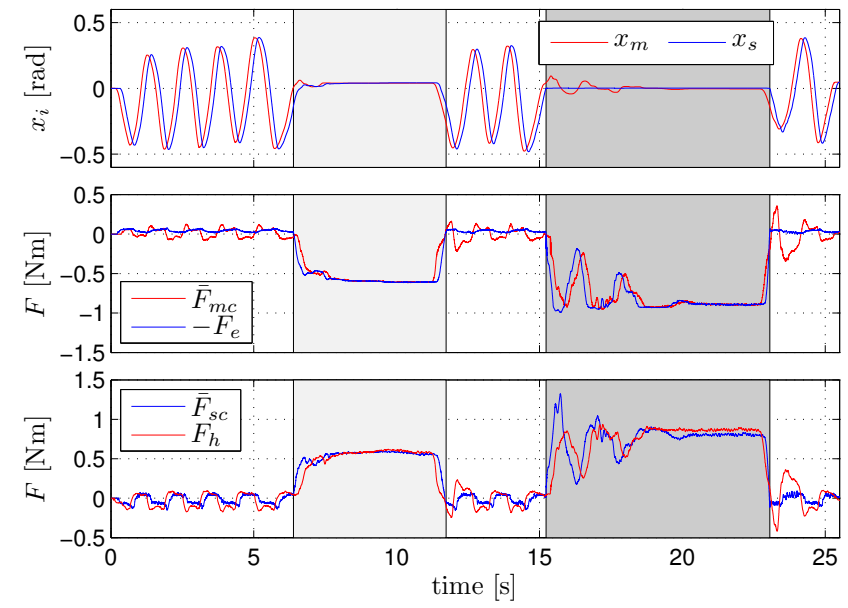

(a) Positions and forces.
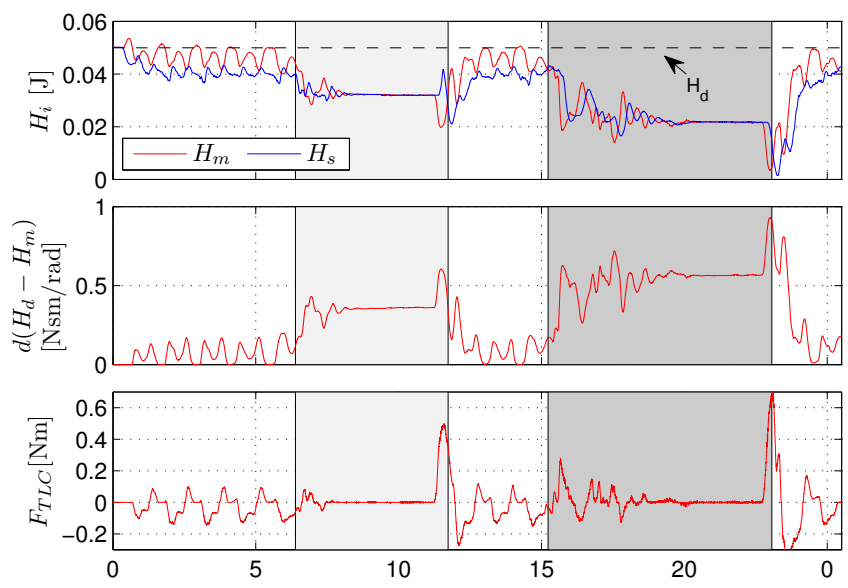

(b) Energy levels $H_{m}$ and $H_{s}$, damping gain $d\left(H_{d}-H_{m}\right)$ and tank level control force $F_{T L C}$.

Figure 8. Experimental results with 2-layer controller of [?] for $T_{m}=T_{s}=$ $0.15 \mathrm{~s}$. In the light grey area the slave is in contact with the spring $\left(x_{e}=-0.1\right.$ $\mathrm{rad})$. In the dark grey area the slave is in contact with the aluminum cylinder $\left(x_{e}=0 \mathrm{rad}\right)$.

2) more severe oscillations during the impact phase that damp out slower and the slave almost breaks contact after the first impact. In ??, using our controller, the oscillations are smaller and damp out faster due to the variable scaling gains $\lambda_{i}$. Such scaling gains are absent in the controller proposed in [?].

3) higher force reflection in steady-state, because with our controller $\lambda_{i}<1$ during steady-state. Both controllers reflect a similar environment stiffness, but with the controller proposed in [?] the aluminum cylinder is perceived at $x_{m}=0 \mathrm{rad}$, whereas with our controller the aluminum cylinder is perceived at $x_{m}=0.05 \mathrm{rad}$ (this is a consequence of the scaling gains $\left.\lambda_{i}\right)$.

4) more damping during the detachment phase, which results in a sticky feeling when detaching (approximately at $11 \mathrm{~s}$ and $23 \mathrm{~s}$ ). Due to the decreasing variable gains $\lambda_{i}$ during the detachment phase, our controller has smaller variable damping gains and these gains converge to zero faster, without causing a sticky feeling.

Summarizing, our proposed controller mainly differs from the one proposed in [?] in the use of two separate energy balances (following the direct force-reflection philosophy) in combination with variable scaling gains $\lambda_{i}$ that are modified automatically when not enough energy can be harvested. This results in significantly less damping during free motion, improved impact and detachment transient responses, at the cost of a reduced force reflection.

\section{CONCLUSION}

In this article, a two-layer control architecture is proposed for direct force-reflecting bilateral teleoperation systems subject to communication delays. In principle, any traditional controller can be implemented in the Performance Layer $(\mathrm{PeL})$, while the Passivity Layer $(\mathrm{PaL})$ guarantees a passive interconnection by adapting the amount of damping injected on the master or slave device and modulating the PeL output when necessary. According to the direct force-reflection philosophy, the design of the PaL is based on a duplication of the power exchanged between the operator/environment and the teleoperator. Passivity of the teleoperator is analyzed and a formal proof is provided, based on the assumption that the operator and environment forces are bounded by an arbitrary large constant and that they do not inject energy when their velocity is bigger than an arbitrary large speed. The experimental results illustrate the performance that can be obtained for a rather large, but realistic, round-trip delay of 300 ms and contact with both a soft spring and a hard aluminum environment. The damping gains are low in free motion, whereas in contact the damping gains increase to stabilize the teleoperator. The force-reflection gains are also reduced during the impact and detachment phase to prevent a recoiling of the master, but their values $\left(\lambda_{m}=0.92\right.$ and $\lambda_{s}=1$ during steady-state contact with the aluminum cylinder) are higher compared to those usually found in literature for any direct force-reflecting controller. Finally, an experimental comparison with the 2-layer controller proposed in [?] revealed that at the cost of a reduced force reflection, our controller requires significantly less damping during free motion and has improved impact and detachment transient responses.

Future work will focus, on the theoretical side, on improving the achievable performance even further. Especially in the contact phase, the goal is to increase the force-reflection gains to obtain the optimal performance computed by the PeL. This could be achieved by optimizing the tuning of the PeL and $\mathrm{PaL}$ parameters and by investigating (nonlinear) alternatives to generate and harvest energy in the PaL. Further extensions should also make the proposed architecture applicable to time-varying delays and data losses in the communication. On the practical side, future research will investigate the effectiveness of the controller on a multi-DOF teleoperator and/or with different master and slave dynamics. For a multiDOF teleoperator, an increase in the force-reflection gains could be achieved by, e.g., harvesting energy from directions tangential to the direction of contact. 


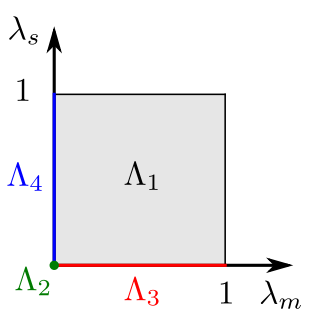

Figure 9. Relation between $\Lambda_{k}, k \in\{1,2,3,4\}$ and the state-dependent, continuously varying gains $\lambda_{m}$ and $\lambda_{s}$.

\section{APPENDIX}

The following Lemma states that $V$, presented in (??), is bounded from below, i.e. $V>V$.

Lemma 1: Given ????, consider the teleoperator described by (??), (??), (??)-(??), (??)-(??) and (??). At the initial time $t_{0}$, let $\left|\dot{x}_{i}\left(t_{0}\right)\right| \leq \overline{\dot{x}}_{i}$ and $\underline{E}_{i, b a l}\left(t_{0}\right)>E_{i, b 2}$, with $E_{i, b 2}$ introduced in ??. Then, the storage functional $V$, defined by (??), satisfies

$$
V>\underline{V}>-\infty,
$$

for all time $t \geq t_{0}$, with $\underline{V}$ defined in (??).

Proof: From ?? and (??) it follows that

$$
\begin{aligned}
& E_{h, \text { com }} \geq-\Delta \bar{E}_{h}, \quad \forall t \in \mathcal{T}, \\
& E_{e, \text { com }} \geq-\Delta \bar{E}_{e}, \quad \forall t \in \mathcal{T},
\end{aligned}
$$

with $\mathcal{T}:=\left[t_{0}, \infty\right)$. Split $\mathcal{T}$ in the intervals

$$
\begin{aligned}
& \mathcal{T}_{1}:=\left\{t \in \mathcal{T} \mid \underline{E}_{m, \text { bal }}>E_{m, b 2} \wedge \underline{E}_{s, \text { bal }}>E_{s, b 2}\right\}, \\
& \mathcal{T}_{2}:=\left\{t \in \mathcal{T} \mid \underline{E}_{m, \text { bal }} \leq E_{m, b 2} \wedge \underline{E}_{s, \text { bal }} \leq E_{s, b 2}\right\}, \\
& \mathcal{T}_{3}:=\left\{t \in \mathcal{T} \mid \underline{E}_{m, \text { bal }}>E_{m, b 2} \wedge \underline{E}_{s, \text { bal }} \leq E_{s, b 2}\right\}, \\
& \mathcal{T}_{4}:=\left\{t \in \mathcal{T} \mid \underline{E}_{m, \text { bal }} \leq E_{m, b 2} \wedge \underline{E}_{s, \text { bal }}>E_{s, b 2}\right\},
\end{aligned}
$$

such that $\mathcal{T}_{1} \cup \mathcal{T}_{2} \cup \mathcal{T}_{3} \cup \mathcal{T}_{4}=\mathcal{T}$. By hypothesis, $t_{0} \in \mathcal{T}_{1}$. Due to the shape of the function $\lambda_{i}$ depicted in ??, $t \in \mathcal{T}_{k} \Longleftrightarrow$ $\left(\lambda_{m}(t), \lambda_{s}(t)\right) \in \Lambda_{k}$, with $k \in\{1,2,3,4\}$ and $\Lambda_{k}$ depicted in ??. To prove (??), we now treat each case $\mathcal{T}_{k}$ separately.

Interval $\mathcal{T}_{1}$ : In this region, $\underline{E}_{i, b a l}>E_{i, b 2}$. From (??), using (??), (??), and $E_{i} \geq 0$, it follows that

$$
V>E_{m, b 2}+E_{s, b 2}-\Delta \bar{E}_{h}-\Delta \bar{E}_{e}, \quad \forall t \in \mathcal{T}_{1} .
$$

Interval $\mathcal{T}_{2}$ : Denote an arbitrary time of entering $\mathcal{T}_{2}$ by $t_{2}>t_{0}$, such that $\underline{E}_{i, b a l}\left(t_{2}\right)=E_{i, b 2}$. Then, with $\underline{E}_{i, b a l} \leq$ $E_{i, b 2}, \forall t \in \mathcal{T}_{2}$, it follows that $\lambda_{i}=0$, such that (??) reduces to

$$
\begin{aligned}
\dot{E}_{m, b a l}+\dot{E}_{e, c o m} & =-F_{e}^{\top} \dot{x}_{s}+F_{m, r e c}^{\top} \dot{x}_{m}, \\
\underline{E}_{s, b a l}+\dot{E}_{h, \text { com }} & =F_{h}^{\top} \dot{x}_{m}+F_{s, r e c}^{\top} \dot{x}_{s} .
\end{aligned}
$$

Summing up (??) and (??), using (??) with $\lambda_{i}=0$, yields

$$
\begin{aligned}
& \underline{\dot{E}}_{m, b a l}+\underline{\dot{E}}_{s, b a l}+\dot{E}_{h, c o m}+\dot{E}_{e, c o m}= \\
& \dot{E}_{m}+\dot{E}_{s}+2 F_{m, r e c}^{\top} \dot{x}_{m}+2 F_{s, r e c}^{\top} \dot{x}_{s} \geq \dot{E}_{m}+\dot{E}_{s},
\end{aligned}
$$

since $F_{i, r e c}^{\top} \dot{x}_{i} \geq 0$. Time integration of both sides of (??) from $t_{2}$ to $t$, recalling that $\underline{E}_{i, b a l}\left(t_{2}\right)=E_{i, b 2}$, yields

$$
\begin{aligned}
\underline{E}_{m, b a l}(t) & +\underline{E}_{s, b a l}(t)+E_{h, c o m}(t)+E_{e, c o m}(t) \\
\geq & E_{m, b 2}+E_{s, b 2}+E_{h, c o m}\left(t_{2}\right)+E_{e, c o m}\left(t_{2}\right) \\
& +E_{m}(t)-E_{m}\left(t_{2}\right)+E_{s}(t)-E_{s}\left(t_{2}\right) \\
\geq & E_{m, b 2}+E_{s, b 2}-\Delta \bar{E}_{h}-\Delta \bar{E}_{e}-\bar{E}_{m}-\bar{E}_{s},
\end{aligned}
$$

where for the last step the bounds (??) and (??) are used. Using the above inequality in (??), recalling that $E_{i, b a l} \geq \underline{E}_{i, b a l}$ due to (??) and $E_{i} \geq 0$ due to (??), gives

$$
V \geq E_{m, b 2}+E_{s, b 2}-\Delta \bar{E}_{h}-\Delta \bar{E}_{e}-\bar{E}_{m}-\bar{E}_{s},
$$

Interval $\mathcal{T}_{3}$ : Denote an arbitrary time of entering $\mathcal{T}_{3}$ by $t_{3}>$ $t_{0}$, such that $\underline{E}_{s, b a l}\left(t_{3}\right)=E_{s, b 2}$ and $\underline{E}_{m, b a l}\left(t_{3}\right)>E_{m, b 2}$. Since in this region $\underline{E}_{s, b a l} \leq E_{s, b 2}$ and $\underline{E}_{m, b a l}>E_{m, b 2}$ for all $t \in \mathcal{T}_{3}$, it follows that $\lambda_{s}=0$ and $\lambda_{m}>0$ for all $t \in \mathcal{T}_{3}$, such that (??) reduces to

$$
\begin{aligned}
\dot{E}_{s, b a l}+\dot{E}_{h, \text { com }}= & F_{h}^{\top} \dot{x}_{m}+F_{s, r e c}^{\top} \dot{x}_{s} \\
& +\lambda_{m}^{T_{m}}\left(F_{m, h a r v}^{T_{m}}\right)^{\top} \dot{x}_{m}^{T_{m}} \\
\dot{E}_{m, \text { bal }}+\dot{E}_{e, c o m}= & -F_{e}^{\top} \dot{x}_{s}+\left(1-\lambda_{m}\right) F_{m, r e c}^{\top} \dot{x}_{m} \\
& -\lambda_{m} F_{m c}^{\top} \dot{x}_{m}-\lambda_{m} P_{m, \text { diss }}
\end{aligned}
$$

To obtain a lower bound on $\underline{E}_{s, b a l}+E_{h, c o m}$, solve (??) for $F_{e}^{\top} \dot{x}_{s}$ and use it in (??). Solve the resulting expression for $\lambda_{m} F_{m c}^{\top} \dot{x}_{m}$ and use it in (??). Then, solve the newly obtained expression for $F_{h}^{\top} \dot{x}_{m}$ and use it in (??) to obtain

$$
\begin{array}{r}
\dot{E}_{s, b a l}+\dot{E}_{h, c o m}=\lambda_{m} F_{m, h a r v}^{\top} \dot{x}_{m}+\lambda_{m}^{T_{m}}\left(F_{m, h a r v}^{T_{m}}\right)^{\top} \dot{x}_{m}^{T_{m}} \\
+\dot{E}_{m}-\dot{E}_{s}+\underline{\dot{E}}_{m, b a l}+\dot{E}_{e, c o m}+\lambda_{m} P_{m, \text { diss }} \\
\geq \dot{E}_{m}-\dot{E}_{s}+\underline{E}_{m, \text { bal }}+\dot{E}_{e, \text { com }}
\end{array}
$$

since by design $P_{m, d i s s} \geq 0$ and $\lambda_{m} F_{m, h a r v}^{\top} \dot{x}_{m} \geq 0$. Time integration of (??) from $t_{3}$ to $t$, together with (??) of ?? (presented below), (??), (??) and (??), gives

$$
\begin{array}{r}
\underline{E}_{s, b a l}(t)+E_{h, \text { com }}(t) \geq \underline{E}_{s, \text { bal }}\left(t_{3}\right)+E_{h, \text { com }}\left(t_{3}\right)+E_{m}(t) \\
-E_{m}\left(t_{3}\right)-E_{s}(t)+E_{s}\left(t_{3}\right)+\underline{E}_{m, b a l}(t)-\underline{E}_{m, b a l}\left(t_{3}\right) \\
+E_{e, c o m}(t)-E_{e, c o m}\left(t_{3}\right) \\
\geq-\left(1+\alpha_{m}\right) \bar{E}_{m}-\bar{E}_{s}+E_{m, b 2}+E_{s, b 2}-E_{m, b 3} \\
-E_{m, \text { diff }}\left(t_{0}\right)-2 \Delta \bar{E}_{e}-\Delta \bar{E}_{h} .
\end{array}
$$

Using this expression in (??), together with $\underline{E}_{m, b a l}>E_{m, b 2}$, $E_{i, b a l} \geq \underline{E}_{i, b a l}$ due to (??), and $E_{i} \geq 0$ due to (??), results in

$$
\begin{aligned}
V> & -\left(1+\alpha_{m}\right) \bar{E}_{m}-\bar{E}_{s}+2 E_{m, b 2}+E_{s, b 2}-E_{m, b 3} \\
& -E_{m, \text { diff }}\left(t_{0}\right)-3 \Delta \bar{E}_{e}-\Delta \bar{E}_{h}, \quad \forall t \in \mathcal{T}_{3} .
\end{aligned}
$$

Interval $\mathcal{T}_{4}$ : By using a similar approach as for region $\mathcal{T}_{3}$, the following result is obtained due to the symmetric design of the PaL

$$
\begin{aligned}
V> & -\bar{E}_{m}-\left(1+\alpha_{s}\right) \bar{E}_{s}+E_{m, b 2}+2 E_{s, b 2}-E_{s, b 3} \\
& -E_{s, \text { diff }}\left(t_{0}\right)-3 \Delta \bar{E}_{h}-\Delta \bar{E}_{e}, \quad \forall t \in \mathcal{T}_{4} .
\end{aligned}
$$


Equation (??) follows directly from the lower bounds (??), (??), (??) and (??) of regions $\mathcal{T}_{1}, \mathcal{T}_{2}, \mathcal{T}_{3}$ and $\mathcal{T}_{4}$.

Lemma 2: Given ????, it follows for all $t \geq t_{0}$ that

$$
\underline{E}_{i, b a l}(t)+E_{i}(t) \leq E_{i, d i f f}\left(t_{0}\right)+E_{i, b 3}+\left(1+\alpha_{i}\right) \bar{E}_{i}
$$

with the constant $E_{i, b 3}$ defined in (??) and the constant $\bar{E}_{i}$ defined in (??).

Proof: Use (??) in (??) to obtain

$$
\begin{aligned}
\dot{E}_{m, \text { diff }}= & F_{h}^{\top} \dot{x}_{m}-\left(F_{e}^{T_{s}}\right)^{\top} \dot{x}_{s}^{T_{s}}-\lambda_{m} F_{m, \text { harv }} \dot{x}_{m} \\
& +P_{m, g e n}-\lambda_{m} P_{m, d i s s}-\dot{E}_{m} \\
\dot{E}_{s, \text { diff }}= & \left(F_{h}^{T_{m}}\right)^{\top} \dot{x}_{m}^{T_{m}}-F_{e}^{\top} \dot{x}_{s}-\lambda_{s} F_{s, \text { harv }} \dot{x}_{s} \\
& +P_{s, \text { gen }}-\lambda_{s} P_{s, \text { diss }}-\dot{E}_{s}
\end{aligned}
$$

From ??, it follows that $F_{h}^{\top} \dot{x}_{m} \leq \bar{F}_{h} \dot{x}_{m}^{a}$ and $-F_{e}^{\top} \dot{x}_{s} \leq$ $\bar{F}_{e} \dot{x}_{s}^{a}$. Moreover, using $0 \leq \lambda_{i} \leq 1$, it follows from (??) and (??) that $P_{i, g e n}-\lambda_{i} P_{i, d i s s} \leq-\alpha_{i} E_{i, d i f f}$. Then, together with $F_{i, h a r v} \dot{x}_{i} \geq 0$, (??) can be bounded as

$$
\dot{E}_{i, d i f f} \leq-\alpha_{i} E_{i, d i f f}+\left(\bar{F}_{h} \dot{x}_{m}^{a}+\bar{F}_{e} \dot{x}_{s}^{a}\right)-\dot{E}_{i} .
$$

By adding and subtracting $\alpha_{i} E_{i}$ and using (??), (??) can be rewritten to

$$
\dot{E}_{i, d i f f}+\dot{E}_{i} \leq-\alpha_{i}\left(E_{i, d i f f}+E_{i}\right)+\alpha_{i} E_{i, b 3}+\alpha_{i} E_{i} .
$$

Using the Comparison Lemma (see [?, Chapter 3]), the following result is obtained

$$
\begin{aligned}
E_{i, d i f f}(t)+E_{i}(t) \leq & e^{-\alpha_{i}\left(t-t_{0}\right)}\left(E_{i, d i f f}\left(t_{0}\right)+E_{i}\left(t_{0}\right)\right) \\
& +\alpha_{i} E_{i, b 3} \int_{t_{0}}^{t} e^{-\alpha_{i}(t-\sigma)} d \sigma \\
& +\int_{t_{0}}^{t} e^{-\alpha_{i}(t-\sigma)} \alpha_{i} E_{i}(\sigma) d \sigma
\end{aligned}
$$

Since $\alpha_{i}>0$ and $0 \leq E_{i}(t) \leq \bar{E}_{i}$ due to (??), it follows that

$$
E_{i, d i f f}(t)+E_{i}(t) \leq E_{i, d i f f}\left(t_{0}\right)+E_{i, b 3}+\left(1+\alpha_{i}\right) \bar{E}_{i} .
$$

Then, since the last two terms in (??) are nonpositive by design, it follows that $\underline{E}_{i, b a l} \leq E_{i, d i f f}$. Using this result, together with (??), we finally obtain (??).

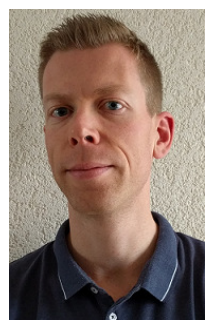

Dennis Heck received his M.Sc degree in Mechanical Engineering at the Eindhoven University of Technology, the Netherlands. His master thesis research focussed on motion and force control for cooperative manipulation under the supervision of Prof. H. Nijmeijer. In 2015, he obtained his Ph.D. degree at the Dynamics and Control group of the Eindhoven University of Technology, under the supervision of prof.dr. H. Nijmeijer and dr. A. Saccon. His research, which was supported by STW and part of the H-Haptics project, focused on the controller design for delayed bilateral teleoperation. Since 2015, he works as a thermal control engineer in the high-tech semiconductor industry. His recent work involves the control of input non-affine Peltier elements and the control of complex underactuated and undersensed thermo-mechanical systems.

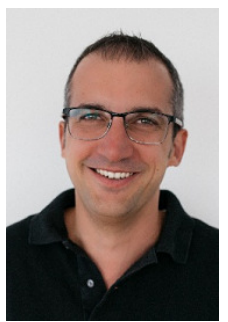

Alessandro Saccon received the laurea degree cum laude in computer engineering and the $\mathrm{Ph} . \mathrm{D}$. degree in control system theory from the University of Padova, Italy, in 2002 and 2006. His thesis received the Claudio Maffezzoni best PhD thesis award by the Politecnico di Milano. After the completion of his $\mathrm{PhD}$ studies, he held until 2009 a research and development position at University of Padova in joint collaboration with Ducati Corse working on control and optimization methods for the exploration of the dynamics of racing motorcycles for virtual prototyping studies using multi-body models and numerical optimal control methods. From 2009 until 2012, he held a post-doctoral research position at the Instituto Superior Técnico, Lisbon, Portugal, sponsored by the Portuguese Science and Technology Foundation (FCT), working on motion planning, dynamics, and control of autonomous robotic vehicles. Since 2013, he is an Assistant Professor on nonlinear control and robotics at the Department of Mechanical Engineering, Eindhoven University of Technology, the Netherlands. His areas of expertise include modeling, analysis, and control of multi-body systems, geometric mechanics, nonlinear control theory, and numerical optimal control for exploration of trajectory space of complex and highly manoeuvrable nonlinear systems. Recent work has focused on the development of optimal constrained motion planning strategies for multiple autonomous robotic vehicles, robotics systems with unilateral position constraints, and control of mechanical systems subject to rigid contacts and impacts.

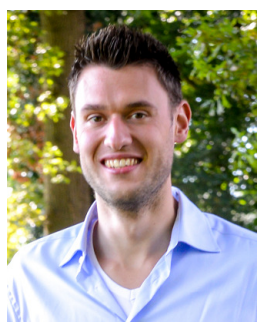

Ruud Beerens (1988) received his M.Sc. degree in Mechanical Engineering at the Eindhoven University of Technology, Eindhoven, the Netherlands, in 2015. His master thesis research focused on control of bilateral teleoperation systems. He is currently pursuing the Ph.D. degree at the Department of Mechanical Engineering, Eindhoven University of Technology, where his current research focus is on nonlinear and hybrid control design for highperformance mechatronic systems.

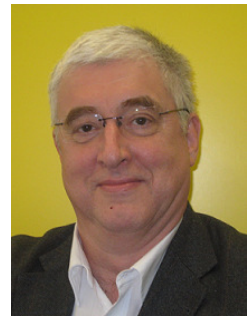

Henk Nijmeijer (1955) is a full professor at Eindhoven, and chairs the Dynamics and Control group. He has published a large number of journal and conference papers, and several books, and is or was at the editorial board of numerous journals. He is an editor of Communications in Nonlinear Science and Numerical Simulations. He is a fellow of the IEEE since 2000 and was awarded in 1990 the IEE Heaviside premium. He is appointed honorary knight of the 'golden feedback loop' (NTNU) in 2011. He is since 2011 an IFAC Council Member. Per January 2015 he is scientific director of the Dutch Institute of Systems and Control (DISC). He is recipient of the 2015 IEEE Control Systems Technology Award. He is program leader of the Dutch research program 'Integrated Cooperative Automated Vehicles' (i-CAVE). Since April 2017 he is director of the Graduate Program Automotive Technology of the Eindhoven University of Technology. 